\title{
Sleep apnoea in ischaemic heart disease: differences between acute and chronic coronary syndromes
}

\author{
P Moruzzi, S Sarzi-Braga, M Rossi, M Contini
}

\begin{abstract}
Objective-To evaluate the incidence of sleep apnoea in acute and chronic coronary syndromes.

Design-Analysis of sleep and breathing characteristics in a polysomnographic study.

Setting-Cardiology department in tertiary referral centre.

Patients-23 patients were studied soon after acute myocardial infarction (group 1), 22 after clinical stabilisation of unstable angina (group 2), and 22 who had stable angina (group 3). Conditions liable to cause sleep apnoea, such as obesity, chronic obstructive pulmonary disease, neurological disorders, or the use of benzodiazepines, were exclusion criteria. Main outcome measures-Sleep apnoea and hypopnoea, oxygen saturation, and sleep indices evaluated soon after clinical stabilisation in groups 1 and 2 and also in group 3.
\end{abstract}

Results-Sleep apnoea, mainly of the central type, was equally present in groups 1 and 2 (mean (SD) apnoea-hypopnoea index: $11.10(19.42)$ and 14.79 (20.52), respectively) and more severe than in group 3 (2.82 (6.43), p < 0.01). Total time spent at $\mathrm{SaO}_{2}<90 \%$, although significantly greater in group 1 and $2(0.89$ (2.4), $1.42(3.23) \mathrm{min})$ than in group $3(0.01$ $(0.05) \min , \mathrm{p}<0.05)$, was clinically irrelevant. More arousals per hour of sleep $(p<0.05)$ were detected in group $1(5.15$ (3.71)) and group 2 (5.31 (2.14)) than in group 3 (2.83 (1.51)).

Conclusions-Sleep apnoea, chiefly of the central type, not only characterises acute myocardial infarction, as found by others, but also unstable angina studied after recent stabilisation. Patient selection by exclusion of other causes of breathing disorders shows that coronary disease related apnoea is absent in the chronic coronary syndrome. In acute syndromes the lack of clinically significant apnoea related oxygen desaturation, together with the low associated incidence of major ischaemic and arrhythmic events, suggests that sleep apnoea is benign in these circumstances, despite a worsening of sleep quality.

(Heart 1999;82:343-347)

Keywords: acute myocardial infarction; unstable angina; stable angina; sleep apnoea
Sleep apnoea may be present in several cardiovascular diseases, such as congestive heart failure, ${ }^{1-4}$ systemic arterial hypertension, ${ }^{5-7}$ and coronary artery disease. ${ }^{8-10}$

According to some investigators, sympathetic activation can induce apnoea. ${ }^{11}{ }^{12}$ If this were the case, increased adrenergic drive might help to explain the prevalence of sleep apnoea in such conditions as congestive heart failure and systemic arterial hypertension, but not in all varieties of coronary artery disease. Thus, while acute myocardial infarction ${ }^{13}{ }^{14}$ and unstable angina ${ }^{15}$ are associated with sympathetic overactivity, chronic coronary artery disease without compromised left ventricular function is not. ${ }^{14}$ On the other hand, it is also possible that apnoea facilitates ischaemic attacks through hypoxia and the ensuing sympathetic activation. ${ }^{16} 17$

In order to try to explain the association between coronary artery disease and sleep apnoea, patient selection must be strict enough to exclude the presence of non-cardiovascular causes of apnoea. Our aim in this study was to evaluate sleep and the breathing patterns during sleep in patients suffering from stable coronary artery disease, recent myocardial infarction, and unstable angina, to determine whether there is a difference between coronary stability and coronary instability in relation to the incidence of sleep apnoea. These conditions may differ not only because of different degrees of sympathetic activation, but also with regard to other characteristics such as left ventricular function and thrombogenesis, which might influence or be influenced by sleep apnoea. In stable coronary artery disease, for example, left ventricular function and thrombogenesis are often normal $^{18}$; in unstable angina, an increase in thrombogenesis contributes to clinical instability, ${ }^{19}{ }^{20}$ while ventricular function may be normal, at least at baseline; and in myocardial infarction there is often an associated reduction in left ventricular function and enhanced thrombogenesis. ${ }^{121}$

We thought that evaluating the prevalence of sleep apnoea and its relation to sleep stages under these different conditions might help in separating cause from effect with regard to breathing alterations in coronary artery disease. In particular, we wanted to see whether there is a link between coronary disorders associated with sympathetic activation and sleep apnoea.

Methods

We studied 67 patients (56 men and 11 women), ranging in age from 32 to 70 years, mean (SD) 
Table 1 Baseline characteristics of the patients

\begin{tabular}{llll}
\hline & $\begin{array}{l}\text { Group 1 } \\
\text { (myocardial infarction) }\end{array}$ & $\begin{array}{l}\text { Group 2 } \\
\text { (unstable angina) }\end{array}$ & $\begin{array}{l}\text { Group 3 } \\
\text { (stable angina) }\end{array}$ \\
\hline Age (years) & $56.00(7.53)$ & $54.45(9.97)$ & $57.10(8.61)$ \\
Sex (M/F) & $22 / 1$ & $16 / 6$ & $18 / 4$ \\
BMI (kg/m) & $25.76(3.33)$ & $24.56(3.15)$ & $26.69(3.05)$ \\
Ejection fraction (\%) & $53.04(11.17) \dagger$ & $62.19(11.91)^{\star}$ & $68.40(8.24)$ \\
Heart rate (beats/min) & $59.2(6.64)$ & $59.38(8.23)$ & $56.0(5.94)$ \\
$\beta$ Blockers & 7 & 9 & 7 \\
Smoker & 19 & 16 & 11 \\
Hypertension & 8 & 12 & 2 \\
Total number in group & 23 & 22 & 22 \\
\hline
\end{tabular}

Values are numbers of patients or mean (SD).

${ }^{\star} \mathrm{p}<0.05 v$ group $3 ; \mathrm{tp}<0.01 v$ group 3 .

$\mathrm{BMI}$, body mass index. and hypopnoea per hour of sleep, total time spent in apnoea-hypopnoea, mean duration of apnoea-hypopnoea, longest apnoea duration, minimum arterial oxygen saturation $\left(\mathrm{SaO}_{2}\right)$ and time spent at $\mathrm{SaO}_{2}$ less than $90 \%$.

Apnoea episodes were considered to be interruptions in air flow lasting for more than 10 seconds; hypopnoea episodes were defined as periods in which there was at least a $50 \%$ reduction in respiratory flow for 10 seconds or more. Apnoea and hypopnoea episodes were classified as central if no chest or abdominal movement was observed, obstructive if chest and abdominal movements occurred during airflow reduction, and mixed when respiratory effort, initially absent, began just before the restoration of airflow. ing from acute myocardial infarction (group 1), 22 from unstable angina (group 2), and 22 had clinically stable angina (group 3). Previous myocardial infarction and independent risk factors for the development of sleep apnoea-such as lung disease, severe obesity, neurological disorders, and use of drugs liable to interfere with central nervous control of respiration, particularly benzodiazepines - were exclusion criteria. Coronary treatment was maintained and use of $\beta$ blockers was accurately accounted for (table 1) and considered in the analysis.

In group 1, extension of the necrosis was evaluated by the creatine kinase (CK) peak. Patients in group 2 were studied soon after clinical stabilisation. In group 3, the diagnosis of ischaemic heart disease was established by exercise stress testing. Coronary angiography was performed whenever necessary (62 patients). All patients gave informed consent for the study, and the research protocol was approved by the local ethics committee on human research.

A polysomnographic study (Somnostar 4100, SensorMedics bv, Bilthoven, Netherlands) was performed in all patients during their stay in hospital. Overnight recording included a four channel electroencephalogram (EEG) (C4A1, O2A1, C3A2, and O1A2), an electro-oculogram, a submental electromyogram, a three channel ECG, and evaluation of oro-nasal airflow by a thermistor, rib cage and abdominal movements by strain gauges, and oxygen saturation by digital oximetry (SensorMedics)

The day before the study, an echocardiogram, to evaluate left ventricular ejection fraction, and a spirometric test, to exclude the presence of any restrictive or obstructive limitation of respiratory function, were performed in all cases. Patients with abnormal spirometric tests were not included in the study.

The indices used to assess sleep were total sleep time, sleep latency, sleep efficiency (total sleep time $\times 100 /$ time in bed), number of arousals per hour of sleep, and evaluation of sleep stages as percentages of total sleep time. Sleep stages were identified blind by two observers through non-computer analysis, according to the guidelines established by the UCLA brain information service committee. ${ }^{22}$ To evaluate breathing disorders we included in the analysis the number of episodes of apnoea

\section{STATISTICS}

Data are expressed as mean (SD). Statistical analysis was performed by $\chi^{2}$ analysis and Kruskal and Wallis analysis of variance; in case of statistical significance, comparisons between two groups were performed using the MannWhitney $U$ test. Statistical significance was assumed at $\mathrm{p}<0.05$.

\section{Results}

As shown in table 1, the three groups were comparable for age, sex, and body mass index. Patients in groups 1 and 2 were more likely to have hypertension. Smoking and the use of $\beta$ blockers were uniformly distributed.

In group 1 the mean interval since myocardial infarction was 6.9 (3.6) days (range 2 to 14 days). The site of infarction was anterior or anterolateral in 12 cases and inferior or inferoposterior in 11 . Mean peak CK value was 1584 (1159) U/1, ranging from 320 to $5054 \mathrm{U} / 1$. In group 2 at the time of the study, patients had been asymptomatic for $2.27(0.83)$ days (range 1 to 4 days).

Left ventricular ejection fraction was within nificantly less in groups 1 and 2 than in group 3 (table 1). At coronary angiography, single vessel disease was predominant in each group (65\% in group $1,50 \%$ in group 2 , and $47 \%$ in group 3), while two vessel disease was present in $22 \%$ in groups 1 and 2 and $29 \%$ in group 3. Three patients in group 1 (13\%), three patients in group $3(17 \%)$, and five patients in group 2 $(22 \%)$ had three vessel disease. Differences among groups were not statistically significant. No correlation was found between the apnoea-hypopnoea index and the number of coronary arteries involved or the site of myocardial ischaemia or infarction.

Sleep indices and respiratory events are summarised in table 2 . We found no differences among the groups in sleep latency, sleep efficiency, and total sleep time. The number of arousals per hour of sleep was significantly higher in groups 1 and 2 than in group 3. In group 1 and group $2,49 \%$ and $45 \%$ of arousals were preceded by apnoea, respectively.

There was a trend towards lighter sleep (higher percentage of stages 1 and 2 of non-REM sleep) in groups 1 and 2 compared with group 3, but these differences were not normal limits in all groups, although it was sig- 
Table 2 Sleep indices, respiratory events, and oxygen saturation

\begin{tabular}{llll}
\hline & Group 1 & Group 2 & Group 3 \\
\hline Total sleep time (min) & $329(33)$ & $310(68)$ & $328(33)$ \\
Sleep latency (min) & $16.40(11.27)$ & $23.31(14.54)$ & $21.73(16.04)$ \\
Sleep efficiency (\%) & $83.60(7.22)$ & $75.23(17.44)$ & $80.92(10.05)$ \\
Arousals (n/h) & $5.15(3.71)^{\star}$ & $5.31(2.14)^{\star}$ & $2.83(1.51)$ \\
Light sleep (\%) & $71.22(8.94)$ & $72.08(12.87)$ & $67.14(8.53)$ \\
Deep sleep (\%) & $12.45(9.76)$ & $12.10(7.31)$ & $14.23(7.86)$ \\
REM sleep (\%) & $16.05(7.12)$ & $16.63(7.96)$ & $19.45(7.42)$ \\
Sleep heart rate (beats/min) & $57.93(6.49)$ & $57.11(7.15)$ & $54.24(5.85)$ \\
AHI (n/h) & $11.10(19.42) \dagger$ & $14.79(20.52) \dagger$ & $2.82(6.43)$ \\
Mean apnoea duration (s) & $17.64(4.26)^{\star}$ & $16.23(3.63)$ & $13.47(4.73)$ \\
Longest apnoea (s) & $31.56(9.33) \dagger$ & $24.67(10.85)^{\star}$ & $16.95(7.08)$ \\
Apnoea total time (min) & $11.82(12.27) \dagger$ & $8.55(13.88)^{\star}$ & $0.65(0.56)$ \\
Mean SaO 2 (\%) & $96.23(1.12)$ & $96.42(1.35)$ & $97.03(0.69)$ \\
Minimum $\mathrm{SaO}_{2}(\%)$ & $90.10(4.23)^{\star}$ & $89.45(5.24)^{\star}$ & $93.42(2.48)$ \\
Total time at $\mathrm{SaO}_{2}<90 \%(\mathrm{~min})$ & $0.89(2.40)^{\star}$ & $1.42(3.23)^{\star}$ & $0.01(0.05)$ \\
\hline
\end{tabular}

Values are mean (SD).

${ }^{\star} \mathrm{p}<0.05 v$ group $3 ; \mathrm{tp}<0.01 v$ group 3 .

AHI, apnoea-hypopnoea index; REM, rapid eye movement; $\mathrm{SaO}_{2}$, arterial oxygen saturation.

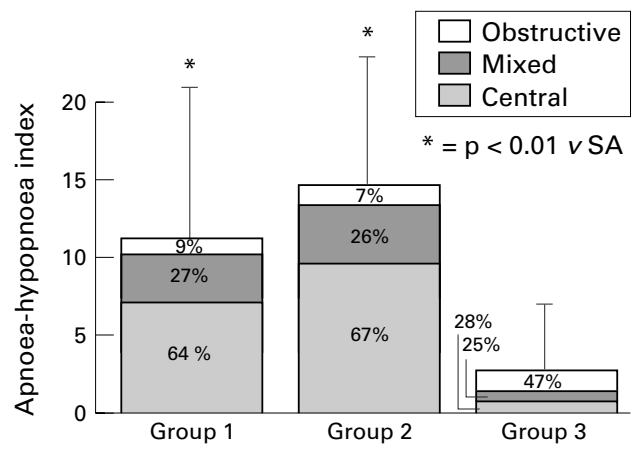

Figure 1 Respiratory events during sleep. Percentages indicate relative distribution of central, mixed, and obstructive events.

statistically significant. The apnoea-hypopnoea index and total sleep apnoea time were significantly increased in groups 1 and 2 compared with group 3. The difference between groups 1 and 2 was not statistically significant. In comparison with group 3 , the mean duration of apnoea episodes was significantly greater only in group 1 patients; statistical significance for group 2 patients was only reached if the analysis was confined to the longest periods of apnoea.

Arterial oxygen saturation was normal at baseline in all patients, with no differences among the groups. Minimum $\mathrm{SaO}_{2}$ during apnoea was significantly lower and time spent at $\mathrm{SaO}_{2}<90 \%$ was significantly greater in groups 1 and 2 than in group 3, though this was not clinically relevant.

A prevalence of the central type of apnoea was detected both in groups 1 and 2 (64\% and

Table 3 Respiratory variables in subgroups with apnoea-hypopnoea index $(A H I)>10$

\begin{tabular}{|c|c|c|c|}
\hline & Group 1 & Group 2 & Group 3 \\
\hline $\mathrm{AHI}(\mathrm{n} / \mathrm{h})$ & $36.88(31.44)$ & $36.37(20.51)$ & $20.31(11.64)$ \\
\hline Central AHI (n/h) & $26.70(29.55)$ & $23.68(18.54)$ & $4.21(5.69)$ \\
\hline Mixed AHI $(\mathrm{n} / \mathrm{h})$ & $10.70(9.63)$ & $9.85(5.44)$ & $4.66(1.16)$ \\
\hline Obstructive AHI (n/h) & $1.07(1.08)$ & $2.84(4.37)$ & $11.43(16.17)$ \\
\hline Mean apnoea duration (s) & $21.25(3.77)$ & $17.43(3.54)$ & $14.55(0.49)$ \\
\hline Longest apnoea (s) & $37.42(3.12)$ & $45.65(19.39)$ & $27.20(0.14)$ \\
\hline Apnoea total time (min) & $73.57(69.25)$ & $53.41(34.91)$ & $27.18(16.35)$ \\
\hline Mean $\mathrm{SaO}_{2}(\%)$ & $96.0(0.82)$ & $96.0(1.07)$ & $96.5(0.71)$ \\
\hline Minimum SaO $(\%)$ & $86.75(5.50)$ & $85.13(4.39)$ & $90.0(0.02)$ \\
\hline Total time at $\mathrm{SaO}_{2}<90 \%(\mathrm{~min})$ & $0.53(0.67)$ & $3.87(4.54)$ & 0 \\
\hline Total number of patients & 5 & 8 & 2 \\
\hline
\end{tabular}

Values are mean (SD).

$\mathrm{SaO}_{2}$, aterial oxygen saturation.
$67 \%$, respectively), while in group 3 mixed and obstructive apnoea episodes were prevalent (72\%) (fig 1).

These respiratory events were mainly present during light non-REM sleep (77.2 $(25.4) \%)$ and in REM sleep (20.3 (25.5)\%). Only $1.83(4.52) \%$ of apnoea and hypopnoea episodes occurred in deep sleep. No ischaemic ECG changes or arrhythmias preceded or were in any way related to apnoea episodes.

There was no relation between the apnoea-hypopnoea index and left ventricular ejection fraction or the CK peak.

In relation to the possible role of $\beta$ blockers in precipitating sleep apnoea, we compared the apnoea-hypopnoea index in treated and untreated patients. The index was higher, but not significantly so, in untreated than in treated patients, at $13.06(20.52) v 3.4$ (4.73) (NS). Considering only groups 1 and 2, the apnoea-hypopnoea index was significantly higher in patients not taking $\beta$ blockers $(17.62$ (23.18) v 4.36 (5.4), $\mathrm{p}=0.03)$. Subanalysis showed no association, in our population, between sleep apnoea and hypertension, smoking, body mass index, or age.

Assuming an apnoea-hypopnoea index of $\geqslant 10$ to be a reasonable cut off value, we also identified patients with clinically significant sleep apnoea syndrome. In groups 1 and 2, five $(22 \%)$ and eight $(36 \%)$ patients, respectively, had an apnoea-hypopnoea index of $\geqslant 10$. In group 3 an index of $>10$ was detected in only two patients (11\%). Breathing and oxygen saturation data in these subgroups are summarised in table 3. Apnoea attacks were mostly of central type in groups 1 and 2, while there was a prevalence of obstructive type in group 3.

Even when we considered only those patients with an apnoea-hypopnoea index of $<10$, the mean value was significantly higher in group 1 (3.95 (2.46)) and group 2 (2.46 (1.83)) than in group $3(0.96(0.89)), \mathrm{p}<0.05$.

\section{Discussion}

The well known association between sleep apnoea syndrome and acute myocardial infarction $^{910}$ is not only confirmed but reinforced by our results. In previous reports, patients were studied at variable times after the acute event (from some days to six months), ${ }^{9}$ or no exclusion criteria were used, and the myocardial infarction group included old people and patients with cerebrovascular disease, ${ }^{10}$ who are more prone to sleep apnoea. Our patient selection, on the other hand, was strict because of the exclusion of causes of sleep apnoea such as chronic obstructive pulmonary disease, obesity, stroke, and the use of tranquillisers. Moreover, the studies were performed as near as possible to the acute phase of infarction or unstable angina, as soon as clinical stability was achieved. Despite patient selection, we still observed an increased incidence of sleep apnoea in patients with recent myocardial infarction in comparison with chronic coronary artery disease, both in terms of the mean absolute apnoea-hypopnoea index value and the percentage of patients with clinically significant sleep apnoea syndrome (apnoea-hypopnoea 
index $>10$ ). We also observed this nocturnal respiratory pattern for the first time in patients with a recent episode of unstable angina without previous myocardial necrosis. In these subjects the number of apnoea episodes per hour of sleep was even higher than after myocardial infarction, possibly because of the shorter time interval between the acute phase of the disease and the time of the study.

On the other hand, in patients with chronic coronary artery disease without previous episodes of coronary instability of any kind, the apnoea-hypopnoea index was not only less than in the other two study groups but also less than in the general population, ${ }^{23-25}$ probably because of patient selection. Thus sleep apnoea seems to be related in some way not only to myocardial infarction as such but also to the acute coronary syndrome as a whole.

Moreover, if we consider those few stable angina patients with significant sleep breathing disorders, the apnoea episodes were mostly of the obstructive type, as is usually observed in the general population. ${ }^{23-25}$ On the other hand, in groups 1 and 2, a clear prevalence of central sleep apnoea was observed. This could mean that patients with coronary instability not only show a higher incidence of sleep apnoea than patients with coronary artery disease or the general population, but also that such apnoea episodes probably have a different aetiology from episodes in subjects with a stable coronary circulation.

The results of our study do not allow us to draw definitive conclusions about the causes of disordered breathing during sleep in patients with coronary instability. As to a possible role of myocardial dysfunction, congestive heart failure is also characterised by apnoeic episodes, mostly of the central type, ${ }^{26}$ but they are associated with periodic breathing, ${ }^{27}$ which never occurred in our population. Furthermore, the absence of any correlation between the apnoea-hypopnoea index and the left ventricular ejection fraction, which was within normal limits in all three groups of patients, rules this out as a cause of the sleep apnoea syndrome. In addition, ischaemic or arrhythmic events during sleep that are apt to lead to left ventricular failure never occurred in our study.

Subgroup analysis also excludes an apnoeic effect of pharmacological treatment, especially of $\beta$ blockade, in our population.

In our opinion, the prevalence of central apnoea episodes in groups 1 and 2 is more in favour of defective central ventilatory control. Chemoreceptor dysfunction does not seem to provide the best explanation, with normal cardiac performance and no episodes of CheyneStokes respiration. As sympathetic hyperactivity is a feature common to both the postinfarction period and unstable angina, ${ }^{13} 15$ we hypothesise a possible inhibitory effect of sympathetic tone on respiratory drive in these patients. This effect has been demonstrated in animal studies by catecholamine infusion into the carotid artery ${ }^{12}$ and pacing of the vasomotor centres. ${ }^{11}$ The apparently protective effect of $\beta$ blockers against apnoea observed in groups 1 and 2 is consistent with this hypothesis. Stress condition is another characteristic of coronary instability which might play a role in activating the adrenergic nervous system. ${ }^{28}$

We cannot exclude the possibility that apnoea - as suggested by others ${ }^{9}$ - could facilitate coronary thrombosis and instability through hypoxia and platelet activation, both before and after an acute ischaemic event. ${ }^{29} 30$ In our opinion, however, this is more likely to be the case with obstructive than with central types of apnoea. In fact, obstructive sleep apnoea episodes, being more organic than functional in origin, are likely to be present before the occurrence of coronary events, and above all are usually associated with a noticeably higher degree of oxygen desaturation than observed in our study population. ${ }^{30}$

The mild degree of oxygen desaturation found in our patients, together with the brief mean apnoea duration and normal cardiac function, could explain the lack of clinical complications, either ischaemic or arrhythmic, in patients with recent myocardial infarction and unstable angina. A study carried out in more compromised patients would be of interest in evaluating the clinical importance of sleep apnoea in acute coronary syndromes.

With regard to sleep architecture, the incidence of sleep apnoea episodes in groups 1 and 2 could explain the increased number of arousals, at least in those cases in which there was a clear temporal relation between respiratory and EEG events. However, there was no significant worsening in sleep quality, considered as percentage distribution of sleep stages and sleep efficiency.

\section{CONCLUSIONS}

In subjects without risk factors for obstructive respiratory disorders, acute coronary syndromes-unlike chronic coronary artery disease-are associated with the occurrence of sleep apnoea episodes, mostly of the central type, which is in some way related to the ischaemic event. Sympathetic hyperactivity, which is both associated with such conditions and a cause of apnoeic episodes in animal studies, might explain this finding. The lack of significant complications in apnoeic patients suggests that sleep apnoea is benign in these circumstances, at least when other causes of apnoea are excluded.

1 Yamashiro Y, Kryger MH. Review: sleep in heart failure. Sleep 1993;16:513-23.

2 Hanly PJ, Millar TW, Steljes DG, et al. Respiration and abnormal sleep in patients with congestive heart failure. Chest 1989;96:480-8.

3 Davies SW, John LM, Wedzicha JA, et al. Overnight studies in severe chronic left heart failure: arrhythmias and oxygen desaturation. Br Heart f 1991;65:77-83.

4 Bradley TD, Floras JS. Pathophysiologic and therapeutic implications of sleep apnea in congestive heart failure. $f$ Cardiac Failure 1996;2:223-40.

5 Davies RJ, Belt PJ, Roberts SJ, et al. Arterial blood pressure responses to graded transient arousal from sleep in normal humans. F Appl Physiol 1993;74:1123-30.

6 Guilleminault C, Robinson A. Sleep-disordered breathing and hypertension: past lessons, future directions. Sleep 1997;20:806-11.

7 Stradling J, Davies RJO. Sleep apnea and hypertensionwhat a mess! Sleep 1997;20:789-93.

8 Andreas S, Schulz R, Werner GS, et al. Prevalence of obstructive sleep apnoea in patients with coronary artery disease. Coron Artery Dis 1996;7:541-5. 
9 Hung J, Whitford EG, Parsons RW, et al. Association of sleep apnoea with myocardial infarction in men. Lancet sleep apnoea with

10 Saito T, Yoshikawa T, Sakamoto Y, et al Sleep apnea in patients with acute myocardial infarction. Crit Care Med 1991;19:938-41.

11 Millis RM, Trouth CO, Johnson SM, et al. Arterial oxygen desaturation and respiratory failure associated with sympathetic overactivity. In: Trough CV, Millis RM, KiwullSchone HF, et al, eds. Ventral brainstem mechanisms and control of respiration and blood pressure. New York: Marcel Dekker, 1995:653-62.

12 Pizarro J, Warner MM, Ryan $\mathrm{M}$, et al. Intracarotid norepinephrine infusions inhibit ventilation in goats. Resp Physiol 1992;90:299-310.

13 Vanoli E, Adamson PB, Ba-Lin, et al. Heart rate variability during specific sleep stages. Circulation 1995;91:1918-22.

14 Marchant B, Stevenson R, Vaishnav S, et al. Influence of the autonomic nervous system on circadian patterns of myocardial ischaemia: comparison of stable angina with the early postinfarction period. Br Heart $\mathcal{F}$ 1994;71:329 33 .

15 Huang J, Sopher SM, Leatham E, et al. Heart rate variability depression in patients with unstable angina. Am Heart $\mathcal{F}$ 1995;130:772-9.

16 Franklin KA, Nilsson JB, Sahlin C, et al. Sleep apnoea and nocturnal angina. Lancet 1995;345:1085-7.

17 Schafer H, Koehler U, Ploch T, et al. Sleep-related myocardial ischemia and sleep structure in patients with obstructive sleep apnea and coronary heart disease. Chest 1997;111:387-93.

18 Fitzgerald DJ, Roy L, Catella F, et al. Platelet activation in unstable coronary disease. $N$ Engl f Med 1986;315:983-9.

19 Merlini PA, Bauer KA, Oltrona L, et al. Persistent activation of the coagulation mechanism in unstable angina and myocardial infarction. Circulation 1994;90:61-8.
20 Theroux P, Latour JG, Lether-Gauthier C, et al. Fibrinopeptide $\mathrm{A}$ and platelet factor level in unstable angina pectoris. culation 1987;75:156-62.

21 Eisenberg PR, Sherman LA, Schectman K, et al. Fibrinopeptide A: a marker of acute coronary thrombosis. Circulation 1985;71:912-18.

22 Rechtschaffen A, Kales A, eds. A manual of standardized terminology, techniques and scoring system for sleep stages of human subjects. NIH publication No 204. Bethesda: National Institute of Neurological Disease and Blindness, 1968.

23 Young T, Palta M, Dempsey J, et al. The occurrence of sleep disordered breathing among middle aged adults. $N$ Engl $f$ Med 1993;328:1230-5.

24 Phillips BA, Berry TDR, Schmitt FA, et al. Sleep-disordered breathing in healthy aged persons: two and three year follow up. Sleep 1994;17:411-15.

25 Ohayon MM, Guilleminault C, Paiva T, et al. An international study on sleep disorders in the general population: methodological aspects of the use of the SleepEVAL system. Sleep 1997;20:1086-92

26 White DP. Central sleep apnea. Med Clin North Am 1985;69:1205-19.

27 Lofaso F, Verschueren P, Dubois Rande JL, et al. Prevalence of sleep disordered breathing in patients on a heart transplant waiting list. Chest 1994;106:1689-94.

28 Verrier RL, Dickerson LW. Autonomic nervous system and coronary blood flow changes related to emotional activation and sleep. Circulation 1991;83(suppl II):II-81-9.

29 Rangemark C, Hedner JA, Carlson JT, et al. Platelet function and fibrinolytic activity in hypertensive and normotensive sleep apnea patients. Sleep 1995;18:188-94.

30 Bokinsky G, Miller M, Ault K, et al. Spontaneous platelet activation and aggregation during obstructive sleep apnea and its response to therapy with nasal continuous positive airway pressure. Chest 1995;108:625-30. 